An. MED INTERNA (Madrid) Vol. 19, N. $^{\circ} 4$, pp. $189-191,2002$

\title{
Toxicidad ocular por antimaláricos
}

\author{
J. GRAÑA GIL, M. CABANA VÁZQUEZ***, A. VÁZQUEZ GONZÁLEZ*, \\ M. O. SÁNCHEZ MEIZOSO** \\ Servicio de Reumatología. *Medicina Familiar y Comunitaria. **Servicio de \\ Codificación. ***Servico de Oftalmología. Complejo Hospitalario Juan Canalejo. A \\ Coruña
}

OCULAR TOXICITY OF ANTIMALARIAL DRUGS

\section{RESUMEN}

En este artículo exponemos la evolución de una paciente afectada de Lupus eritematoso sistémico con expresión mucocutánea, hematológica, articular y renal. Debido a la afectación mucocutánea la paciente recibió tratamiento con cloroquina e hidroxicloroquina, presentando toxicidad ocular severa secundaria a antimaláricos.

En este trabajo tratamos de hacer una revisión sobre el uso de antimaláricos en reumatología teniendo en cuenta su utilidad, dosis, efectos secundarios y factores de riesgo tales como dosis acumulada, dosis diaria por peso ideal, duración del tratamiento y función renal, así como de las recomendaciones de revisión oftalmológica en los pacientes tratados.

PALABRAS CLAVE: Hidroxicloroquina. Cloroquina. Dosis. Toxicidad ocular. Factores de riesgo y Revisión Oftalmológica.

\section{ABSTRACT}

We relate the evolution of a patient with Systemic Lupus Erythema tosus with mucocutaneous, hematological, joint and renal expression. The skin lesions were treated with Chloroquine and Hydroxichloroquine and severe ocular toxicity related with antimalarials agents was develo ped.

We revised the antimalarials use in Rheumatology, their utility, doses, adverse events and risk factors as cumulate doses, ideal daily doses, renal function and ophtalmological reviews recomendations.

KEY WORDS: Hydroxychloroquine. Chloroquine. Doses. Ocular toxi city, Risks factors. Ophtalmological reviews.

\section{INTRODUCCIÓN}

Los antipalúdicos siguen siendo el tratamiento de elección para las manifestacines cutaneomucosas y articulares leves o moderadas del Lupus Eritematoso sistémico. Son considerados medicamentos seguros y bien tolerados y con escaso riesgo de efectos secundarios. La hidroxicloroquina se considera más segura pero menos eficaz que la cloroquina, si bien, la elección de una u otra sigue siendo tema de debate y depende en general de la experiencia acumulada. Ambas pueden producir toxicidad ocular por depósito corneal y retiniano. Este último produce alteraciones irreversibles de la visión y el paciente puede no percibir su presencia de forma temprana por lo que se recomiendan revisiones oftalmológicas periódicas. Presentamos a continuación un caso tratado con ambas drogas que presentó toxicidad ocular grave.

\section{CASO APORTADO}

Se trata de una paciente de 31 años diagnosticada de epilepsia a los 18 años. En Diciembre del año 91 cuando tenía 24 años ingresó en el hospital por un cuadro de fiebre, erupción cutánea y artralgias siendo diagnosticada de LES. Su función renal en ese momento se encontraba en el límite de la normalidad con una creatinina de $1 \mathrm{mg} / \mathrm{dl}$, proteinuria mínima y microhematuria. Analíticamente presentaba una leve anemia, VSG de 33, anticuerpos anticardiolipina, ANA (+) 1/2500, AC anti-DNA (+) 1/320, AC y anti-RNP (+), siendo el resto normal. Durante este ingreso se trató con esteroides, con buena respuesta.

En el año 92 sufrió otro brote lúpico con predominio cutáneo presentando rash facial florido, intensa mucositis orofaríngea, con presencia de úlceras en paladar duro, vulvovaginitis y lesiones de aspecto isquémico en pulpejos de dedos que se trataron en principio con heparina y al alta con antiagregantes. Su función renal seguía dentro de la normalidad y en la biópsia renal se objetivó glomerulo

Trabajo aceptado: 14 de julio de 2000

Correspondencia: Jenaro Graña. Complexo Hospitalario Juan Canalejo. Servicio de Reumatología. Xubias de Arriba, 84.15006 A Coruña. 
nefritis lúpica focal IIIa (3 de 22 glomérulos), con presencia de una semiluna celular circunferencial en uno de ellos y escasa cronicidad sin alteraciones vasculares o túbulointersticiales relevantes. Se trató de nuevo con esteroides, permaneciendo únicamente una marcada tendencia a la alopecia al alta.

En Septiembre de ese mismo año se quedó embarazada tratándose con $10 \mathrm{mg} /$ día de prednisona y antiagregantes. A las 3 semanas del parto sufrió un nuevo brote lúpico a pesar de que se había subido la dosis de prednisona a $30 \mathrm{mg} /$ día tras el parto.

En Abril de 94 se inicia tratamiento con cloroquina (Resochin) $250 \mathrm{mg} /$ día, debido a la afectación cutánea, por lo que se envió a la paciente para examen oftalmológico, realizándose test de colores, agudeza visual (10/10 en AO), presión intraocular, fondo de ojo y examen con lampara de hendidura que fue normal, realizándose nueva revisión a los 3 meses y posteriores cada 6 meses.

En el examen oftalmológico realizado en Noviembre del 97 presenta una retracción periférica del campo nasal de ambos ojos, siendo el resto de la exploración ocular normal, incluyendo el test de colores y fondo de ojo. A pesar de que este hallazgo no se atribuyó claramente al tratamiento con cloroquina; ésta fue sustituida por hidroxocloroquina $200 \mathrm{mg} /$ día en Enero del 98.

Cinco meses después la paciente se queja de visión borrosa. En el examen ocular se aprecian las siguientes anomalías: Agudeza visual OD 7/10, OI 8/10. En la lámpara de hendidura se evidencian depósitos subepiteliales corneales en ambos ojos. Por oftalmoscopia se detectó una estenosis vascular y una alteración pigmentaria a nivel macular, más evidente en el ojo derecho. El test de colores reveló una alteración del eje tritán (azul-amarillo). En el estudio campimétrico se apreció retracción muy importante del campo visual, quedando reducido a un islote paracentral inferior en ambos ojos (Fig 1). En este momento a la paciente se le diagnostica una retinopatía por antimaláricos y se suspende el tratamiento. En la angiografía aparece un patrón moteado hiperfluorescente (efecto ventana) no sólo a nivel perifoveolar sino también de forma difusa en toda la retina (Fig2) La realización de potenciales evocados y de una resonancia nuclear magnética, en la que aparecen lesiones subcorticales hiperintensas en T2, descartó una neuritis óptica.

En la última revisión ocular realizada en Diciembre del 98 no se apreciaron variaciones con respecto a la exploración anterior.

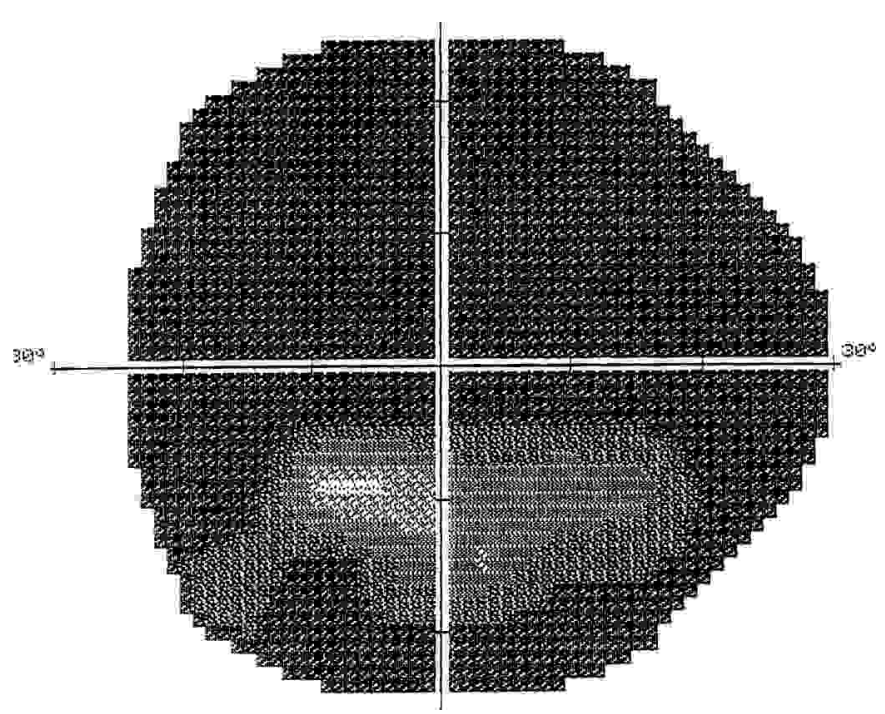

Fig. 1. Campimetría: reducción importante del campo visual, quedando reducido a islote paracentral inferior. Similar en ambos ojos.

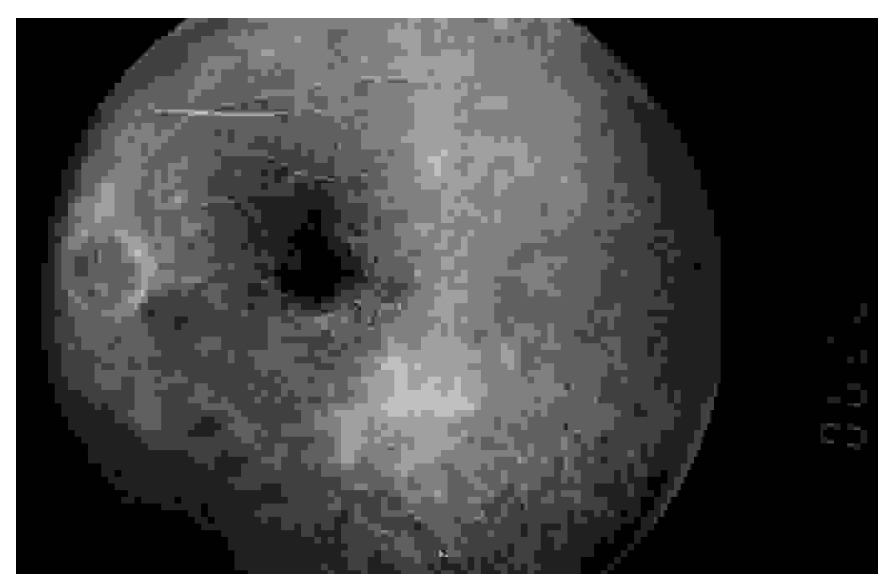

Fig. 2. Angiografía fluoresceínica: patrón moteado hiperfluorescente retiniano difuso. Similar en ambos ojos.

\section{DISCUSIÓN}

El uso de antipalúdicos se ha generalizado en los últimos 20 años para el tratamiento de lupus eritematoso sistémico, discoide, artritis reumatoide y otras enfermedades autoinmunes debido a su escasa incidencia de efectos indeseables. (1)

Son útiles en el lupus para tratar las úlceras orales, poliartralgias, alopecia y serositis leve, disminuyendo su actividad gracias a su efecto antiinflamatorio, complementando a otros AINEs y facilitando la disminución de la dosis de corticoides (2).

Sus efectos secundarios son nauseas, vómitos, lesiones hiperpigmentadas (región pretibial, lecho subungueal y paladar duro). El más grave de sus efectos secundarios es la toxicidad ocular. El depósito corneal del tóxico es uno de los más frecuentes, siendo observado con el doble de frecuencia cuando se trata de cloroquina, que en el caso de hidroxicloroquina $(3,4)$. El depósito corneal también se puede observar con otro tipo de fármacos como la amiodarona (5) y en ningún caso produce síntomas oculares.

En cuanto a la retinopatía, tanto la cloroquina como la hidroxicloroquina son capaces de provocar maculopatía irreversible. Estos efectos son por lo general poco frecuentes según las diversas series. En la de Levy y cols. de 1207 pacientes sólo en 1 caso se demostró retinopatía por hidroxicloroquina $(0,08 \%)$ (6). La hidroxicloroquina es más segura que la cloroquina con una incidencia de retinotoxicidad menor, por otro lado en caso de provocar una lesión retiniana ésta suele ser leve y no progresiva. Esto concuerda con estudios previos, aunque hay que tener en cuenta la mayor frecuencia de utilización de cloroquina y las altas dosis utilizadas hasta hace unas décadas (3).

La toxicidad retiniana se debe a la acumulación del tóxico a nivel intracelular en células ganglionares, fotorreceptores y en particular a nivel del epitelio pigmentario, que puede actuar como reservorio una vez suspendido el tratamiento, manteniendo de esta forma la acción tóxica del fármaco. Los cambios más severos se producen a nivel de las células ganglionares. A nivel histopatológico es característica la despigmentación del epitelio pigmentario de la retina, la pérdida de conos y bastones y la concentración de pigmento intrarretiniano.

Las alteraciones más frecuentes aparecen en los $10^{\circ}$ del área parafoveal (1), dando lugar a la llamada maculopatía en "ojo de buey "que angiográficamente se muestra como un área hiperfluorescente que corresponde al área de atrofia del epitelio pigmentario. Un hallazgo precoz de toxicidad por cloroquina es la detección de pequeños escotomas paracentrales a la luz roja (7). 
Los factores de riesgo señalados en las distintas series son: - La dosis acumulada, que en el caso de nuestra paciente era de $400 \mathrm{~g}$ de cloroquina y de 33,6 $\mathrm{g}$ de hidroxicloroquina.

- La dosis diaria por peso ideal, que en el caso de nuestra paciente, que pesa $66 \mathrm{~kg}$, era de $3,78 \mathrm{mg} / \mathrm{kg} /$ día de cloroquina excepto durante 8 meses en que fue de $7,57 \mathrm{mg} / \mathrm{kg} /$ día, y de $3,03 \mathrm{mg} / \mathrm{kg} / \mathrm{día}$ de hidroxicloroquina sulfato. La dosis óptima según las estudios previos es de $6,5 \mathrm{mg} / \mathrm{kg} /$ día para hidroxicloroquina y menor de $4 \mathrm{mg} / \mathrm{kg} /$ día de cloroquina. $(2,3,7)$

- La duración del tratamiento, de 4 años para nuestra paciente. Se observa mayor riesgo a partir de los 10 años de tratamiento con hidroxicloroquina y de 4 años para cloroquina (7).

-Una función renal conservada es importante ya que en el caso de la hidroxicloroquina se excreta en un $60 \%$ por el riñón, por lo que en caso de insuficiencia renal se aumenta la exposición al tóxico (1).

En el caso de la cloroquina parece que el factor de riesgo más importante es la dosis acumulada (3). La maculopatía necesitaría dosis de 100-300 g de cloroquina para desarrollarse, lo que supondría que los pacientes tratados ininterrumpidamente con cloroquina desarrollarían la toxicidad entre los 2 y los 4 años de tratamiento (7). Mientras en el caso de la hidroxicloroquina el factor de riesgo más importante es el la dosis por peso que no debe superar los $6,5 \mathrm{mg} / \mathrm{kg}$ peso ideal/día.

Otro factor de riesgo encontrado en algún estudio es la asociación de retinopatía y lupus eritematoso sistémico, mayor que con otras enfermedades, lo que sugeriría susceptibilidad subyacente en la enfermedad. En el mismo trabajo se observó a pacientes que inicialmente recibieron terapias prolongadas con cloroquina y posteriormente desarrollaron retinopatía mientras eran tratados con hidroxicloroquina. Esto puede ser debido bien al retraso en la aparición del daño provocado por la primera droga, o bien debido al agravamiento causado por la hidroxicloroquina del daño subyacente que había provocado la cloroquina (4). En nuestro caso, la retinopatía se detectó durante el tratamiento con hidroxicloroquina, pero la toxicidad muy probablemente comenzó con la cloroquina, responsable con seguridad de la retracción inicial del campo visual nasal bilateral.

La recomendación de revisión oftalmológica rutinaria es variable según los autores. En el caso de hidroxicloroquina muchos autores indican que si la dosis es menor de $6,5 \mathrm{mg} / \mathrm{kg} /$ día, la duración del tratamiento es menor de 10 años y la función renal es normal, no sería necesaria la revisión rutinaria. En caso de no ser así, una revisión anual sería adecuada (6). En la serie de Grierson y cols. según el College of Ophthalmologists se recomienda que en pacientes tratados con hidroxicloroquina cuya dosis diaria es menor de $6,5 \mathrm{mg} / \mathrm{kg} /$ día y cuya dosis acumulada es menor de $200 \mathrm{~g}$ no se requiere examen rutinario (5). Otras series indican que en caso de tratamiento con antipalúdicos en las dosis preestablecidas es recomendable una revisión al inicio y después anual a partir del tercer año de tratamiento (8). En cuanto a las revisiones rutinarias los laboratorios recomiendan revisiones cada 3-6 meses y normalmente las recomendaciones médicas indican cada 6 meses (9).

Estas revisiones son de gran importancia dado que si la retinopatía es diagnosticada precozmente puede ser parcial o totalmente reversible en sus primeros estadios. En cuanto a los métodos más frecuentes utilizados para los exámenes rutinarios podemos encontrar: el test de Amsler, la agudeza visual, los perímetros automatizados, el fondo de ojo, la lampara de hendidura, la angiografía y el test de colores. El electrooculograma es para algunos autores la prueba que se afecta con más precocidad (7). Las siguientes en eficacia son el test de colores, los campos visuales y el electroretinograma. Los hallazgos más tardíos son observados mediante oftalmoscopia y angiografía fluoresceínica (7). Hasta la fecha los intentos por encontrar un único test que detecte la toxicidad retiniana en estadíos tempranos y por tanto reversibles, han resultado infructuosos.

Cabe destacar en el caso clínico que presentamos que la primera alteración observada en la paciente fue un escotoma nasal periférico en ambos ojos, hecho poco frecuente con respecto a los escotomas paracentrales, descritos en la mayoría de los casos que aparecen en la literatura. Por otro lado, en la angiografía se evidenció el patrón moteado típico debido a la atrofia del epitelio pigmentario, pero en el caso presentado no sólo aparece en la región perifoveolar sino que se extiende de forma difusa por toda la retina. Además a nivel de las arcadas vasculares se apreció una leve exudación que podría atribuirse no únicamente a su enfermedad sino también a las alteraciones vasculares producidas por la propia toxicidad de los antimaláricos.

Los antipalúdicos aunque con escasos efectos adversos no están exentos de toxicidad, por lo que debemos ser precavidos al utilizarlos.

Siempre es mejor la utilización de hidroxicloroquina debido a su menor toxicidad retiniana.

En caso de utilizar antipalúdicos debemos ser meticulosos en las dosis utilizadas, que deben ser menores de 6,5 $\mathrm{mg} / \mathrm{kg} /$ día para hidroxicloroquina y menor de $4 \mathrm{mg} / \mathrm{kg} /$ día para cloroquina, con tendencia en los últimos trabajos a recomendar dosis de $3 \mathrm{mg} / \mathrm{kg} /$ día. Evitar los tratamientos prolongados que no deberían ser superiores a 10 años para hidroxicloroquina y de 4 años para cloroquina, siendo la dosis acumulada de cloroquina menor de $300 \mathrm{~g}$ y la función renal normal. En este caso la mayoría de los autores indican que el riesgo de toxicidad es mínimo o inexistente. Aun así existen casos descritos de pacientes que incluso cumpliendo estas premisas desarrollaron retinopatía (10), por lo que se debe recomendar revisión oftalmológica rutinaria antes de iniciar el tratamiento y posteriormente cada 6 meses como mínimo.

\section{Bibliografía}

1. Howard N, Bernstein MD. Ocular safety of hydroxychloroquine. Ann opthalmol 1991; 23: 292-296.

2. Rodríguez V, Blanco R, Martínez V. Lupus eritematoso sistémico. Medicine, Tratado de medicina interna. $6^{\text {a }}$ ed. Madrid: ediciones Idepsa, 1992; 17: 789802 .

3. Cox NH, Paterson WD. Ocular toxicity of antimalarials in dermatology. Br J Dermatol 1994; 131: 878-882.

4. Shearer RV, Dubois EL. Ocular changes induced by long-term hydroxychloroquine (plaquenil) therapy.Shearer-RV; Dubois-EL. Am J Ophtalmol 1967; 64: 245-252.

5. Grieson DJ. Hydroxychloroquine and visual screening in rheumatology outpatient clinic. Ann Rheum Dis 1997; 56: 188-190.

6. Levy GD, Munz SJ, Paschal J, Cohen HB, Pince KJ, Peterson T. Incidence of hydroxychloroquine retinopathy in 1207 patients in a large multicenter outpatient practice. Arthritis Rheum 1997; 40: 1482-1486.

7. Corcelles AJ, Minaya F, Morrillo MJ. Maculopatia por cloroquina. Oftalmologia práctica 1997; 6: 44-47.

8. Spalton DJ. Retinopathy and antimalarial drugs the British experience. Lupus 1996; 5 (suppl 1): S70-72.

9. Rynes RI. Ophtalmologic considerations in using antimalarials in the United States. Lupus 1996; 5 (suppl 1): S73-74.

10. Giorgio D, Rosati C, Verrastro G, Grandinetti F. What's the right patient management for early diagnosis of hydroxychloroquine retinal toxicity? Recentigonos Prog Med 1996; 87: 308. 